\title{
Adapting a Community of Practice Model to Design an Innovative Ethics Curriculum in Healthcare
}

\author{
Sudeshni Naidoo ${ }^{a}$ Anthony T. Vernillo ${ }^{b}$ \\ a Department of Community Oral Health, Faculty of Dentistry, University of the Western Cape, Cape Town, \\ South Africa; ${ }^{\mathrm{b}}$ Department of Oral and Maxillofacial Pathology, Radiology and Medicine, New York University \\ College of Dentistry, Center for Bioethics, New York University, New York, N.Y., USA
}

\section{Key Words}

Collaboration · Community of practice $\cdot$ Global health .

Health ethics · Public health · Social capital

\begin{abstract}
The focus of healthcare ethics within the framework of ethical principles and philosophical foundations has always, in recent times, been the community, namely, the healthcare provider, the patient or, in research, the study participant. An initiative is thus described whereby a community of practice (CoP) model was developed around health ethics in health research, education and clinical care. The ethics curriculum was redesigned to include several components that are integrated and all embracing, namely, health research ethics, healthcare ethics, health personnel education in ethics and global and public health ethics. A CoP is a group who share a common interest and a desire to learn from and contribute to the community with their variety of experiences. The CoP is dynamic and organic, generating knowledge that can be translated into effective healthcare delivery and ethical research. It requires the collaboration and social presence of active participants such as community members, healthcare professionals and educators, ethicists and policy makers to benefit the community by developing approaches that adapt to and resonate with the community and its health-
\end{abstract}

care needs. Philosophical principles constitute the foundation or underpinning of this innovative curriculum. Recommendations are presented that will continue to guide the consolidation and sustainability of the CoP.

두 2013 S. Karger AG, Basel

\section{Introduction}

The teaching of ethics, while commonplace in medicine, remains underdeveloped in the rest of the vital, auxiliary health professions such as nursing, occupational therapy, pharmacy, physiotherapy and dentistry. This is particularly true within the developing world. Hence, much of the literature on ethics and law has primarily focused on aspects of either the provision of acute medicine within a hospital setting or clinical research within the same setting [1]. Other issues of topical interest are also well researched but their focus often remains on dramatic medical intervention (e.g. reproductive technologies) or other current aspects of the management of medical provision (e.g. resource allocation) [1].

As reflected by the pattern of their publications, most bioethicists appear to know little about or to be not interested in the practice of the auxiliary professions and related research issues. There are notable exceptions [2, 3],

\begin{tabular}{ll}
\hline KARGER & $\begin{array}{l}\text { (c) 2013 S. Karger AG, Basel } \\
\text { 1011-7571/13/0237-0060\$38.00/0 Operger }\end{array}$ \\
E-Mail karger@karger.com & $\begin{array}{l}\text { This is an Open Access article licensed under the terms of the } \\
\text { www.karger.com/mpp }\end{array}$ \\
$\begin{array}{l}\text { Creative Commons Attribution-NonCommercial 3.0 Un- } \\
\text { ported license (CC BY-NC) (www.karger.com/OA-license), } \\
\text { applicable to the online version of the article only. Distribu- } \\
\text { tion permitted for non-commercial purposes only. }\end{array}$
\end{tabular}

Senior Prof. Sudeshni Naidoo

Faculty of Dentistry

University of the Western Cape, Cape Town, South Africa

Private Bag X1, Tygerberg 7505 (South Africa)

E-Mail suenaidoo@uwc.ac.za 
but they are rare. Equally, there is a paucity of interest among academics in teaching and writing about what has become standard or 'core' ethical and legal components of so many curricula within medical education (e.g. informed consent, confidentiality, truth telling and conformity to treatment). Furthermore, some (but not all) of the provisions of care in the auxiliary professions are elective in nature. Patients are presumed to be treated because they want to be rather than because they have to be. Again, this may seem to lessen the vulnerability of patients and thus the importance of the same levels of attention devoted to ethical and legal education found in medical training. There can be no doubt that this situation is unsatisfactory. Nonetheless, an additional qualification is worth mentioning. Clinical ethics per se is all embracing because it does not apply to physicians alone but to all healthcare providers, for example, nurses, dentists, physiotherapists, pharmacists and others. Against this background, patients or care receivers have the right to refuse treatment offered by these professionals. Autonomy is thus not compromised provided the clinical practice and its practitioners are ethical in the discharge of their duties.

Ethical issues that arise in healthcare and research are generally similar to those so often debated and discussed within medicine [4]. Professionalism and respecting patients' rights are no less important in any other health setting than in a medical clinic. The same moral tensions in medicine between individual rights of various kinds and concerns about provision and research concerning public health are widely represented in other health disciplines such as nursing and dentistry. Nursing and dentistry also have their professional codes and ethical framework derived from bioethical principles. Biomedical ethics has prominence because it is related to the functioning of the healthcare industry generally. However, patients participating in dental and allied health research are just as subject to potential abuse and manipulation as they are in medical research [5]. Again, all of these issues become even more salient in those global communities as regards patients who are disadvantaged, vulnerable, poor and lacking in education. How then could a standard ethics curriculum in the healthcare professions be redesigned to complement a community of practice (CoP), and with such integration to thus ensure effective, ethical healthcare delivery and ethical research practice? This paper describes an exercise where academic faculties from several disciplines (public health, pharmacy, dentistry and bioethics) engaged with the process of exploring the theoretical basis of health ethics and worked together to develop an open-ended and innovative curriculum.

Innovative Ethics Curriculum in Healthcare

\section{What Is a CoP?}

A CoP has been defined as a group who share a common interest and a desire to learn from and contribute to the community with their variety of experiences [6]. Wenger [7] described the structure of a $\mathrm{CoP}$ as consisting of three interrelated terms: mutual engagement, joint enterprise and shared repertoire. Mutual engagement refers to the establishment of norms and the building of collaborative relationships through participation in the community. Joint enterprise is the creation of a shared understanding of what binds them together through their interactions and is (re)negotiated by its members; it is sometimes referred to as the domain of the community. Shared repertoire refers to communal resources that are used in the pursuit of their joint enterprise.

More recently, Wenger [7], and Wenger et al. [8] described learning as 'social participation,' whereby an individual actively participates in the practice of social communities and the construction of identities through these communities. In this regard, a $\mathrm{CoP}$ refers to a group of individuals who participate in communal activities and create a shared identity through engaging in and contributing to the practices of their communities. The structural characteristics of a CoP are then redefined as a domain of knowledge, notion of community and a practice. A domain of knowledge creates common ground, inspires members to participate, guides their learning and gives meaning to their actions. A strong community fosters interactions and encourages a willingness to share ideas. The domain provides the focus and common identity for the community and the practice is around mutual engagement of the community members via participatory interaction [8].

A specific and practical application of the CoP model is in the conduct of health research in countries where access to healthcare and an institutionalized system of delivery are either absent or underdeveloped [5]. Health research is increasingly understood as a partnership between stakeholders to include potential and actual volunteers and their communities. The expectations and obligations of the researchers and participants must be clear from the outset through community engagement, knowledge transfer and management, not only between the researchers as a scientific community but also between the members of the population who self-identify as a separate community. In that regard, the CoP model has practical application in the implementation of a research trial in a developing country. Thus, anticipation and negotiation of any health-related issues concerning the research can reduce sources of possible conflict and define the duties

Med Princ Pract 2014;23(suppl 1):60-68 DOI: $10.1159 / 000353149$ 
and the extent of the obligations of the researchers and sponsors to that population. For example, a clinical trial to test a vaccine against malaria [5] may not be part of the healthcare system but potential participants might want to enrol because it offers them their only opportunity for care. Researchers might be seen by the population as not only able to provide healthcare but as obligated to do so, especially if they are physicians or nurses, and no other healthcare options exist.

CoPs have become associated with finding, sharing, transferring and archiving knowledge [9] and are now viewed by many as a means to capturing the tacit knowledge, or the know-how that is not so easily articulated [10]. Tacit knowledge is essentially knowledge considered to be valuable context-based experiences that cannot be easily captured, codified or stored $[11,12]$. CoPs have become an integral part of the organizational structure in many businesses, institutions and organizations [13]. These communities often take on knowledge of supervising tasks that were formerly covered by more formal institutional structures. In some cases, CoPs are both formal and informal. There is thus a great deal of interest within educational institutions to encourage, support and sponsor the CoP model in order to benefit from shared knowledge that may lead to higher productivity [14].

Knowledge management is thus seen 'primarily as a problem of capturing, organizing and retrieving information, evoking notions of databases, documents, query languages and data mining' [15]. The CoP, collectively and individually, is considered a rich potential source of helpful information in the form of actual experiences or best practices. It is a shared resource of theoretical and practical elements from which to develop and implement an innovative curriculum in an expeditious manner.

\section{Benefits of a CoP}

Social capital is the wealth or benefit that exists within a network of individuals [16] and it is the process of sharing expertise, learning from others, and participating in group activities that members are said to be acquiring [17]. Developing professional relationships and social networks are key reasons as to why people stay within an institution or organization [18]. CoPs have been described as vehicles for generating social capital by developing networks and fostering relationships that build mutual confidence and obligation [19]. Social capital is linked to behaviours such as respect and trust, giving people a sense of belonging within the social entity [8].
Furthermore, there is the benefit of time saved, as members within the community have tacit knowledge that can often be difficult to store and retrieve outside. In a CoP, members openly discuss and brainstorm about their subject of interest and, harnessing new-found capabilities, productive curriculum development can be enhanced. The type of information that is shared and learned in a CoP is boundless [20]. Duguid [21] clarifies the difference between tacit knowledge, or knowing how, and explicit knowledge, or knowing what.

Performing optimally in a profession requires translation of theory into practice. CoPs thus help the individual bridge the gap between knowing what and knowing how [21]. Individuals have thus reported an increase in communication, less dependence on geographic proximity and generation of new knowledge [22]. Communicating with others in a $\mathrm{CoP}$ involves creating social presence. It is believed that social presence affects how likely an individual is to participate in a CoP [23]. Collaboration is essential to ensure that CoPs thrive since they are based on collegial relationships rather than the usual hierarchical management structures and to some extent may assist in bridging the gap between practice and political priorities [24-26]. Sveiby and Simon [27] found that experienced colleagues tended to foster a more collaborative culture, and higher educational levels predicted a tendency to favour collaboration.

\section{Building an Interfaculty, Interdisciplinary CoP}

Several recent initiatives at the University of the Western Cape (UWC), Cape Town, South Africa, have begun to respond to the increasing awareness that the ethical conduct of research needs to be more rigorously addressed. As an institution with strong roots in its surrounding communities, the recognition that academic and research activities have important implications for human rights and empowerment has been implicit. However, the intellectual basis upon which to make good ethical choices in research and teaching has not been adequately understood within the UWC health-related disciplines. Even the most progressive university mission statements and research ethics policies are not enough to ensure that the rights and interests of community members are adequately protected from the growing number of health research projects they encounter. The seemingly sisyphean task then was not only how to integrate teaching, clinical care and health research together within a human rights framework as embodied by the Health
Naidoo/Vernillo 
Professions Council of South Africa (HPCSA) requirements [28] but also to build up a CoP around health ethics that was broad-based and sustainable.

CoPs are groups of people who share a concern or a passion for something they do and learn how to do it better as they interact regularly [8]. Practice is described as the result of collective learning and is reflective of social relationships and shared community endeavours [7]. Practical knowledge is related to what we know, what we do and how we act [29]. A systematic review on the use of CoPs in business and healthcare observed that while specific structures of groups varied widely, they shared four characteristics: social interaction among members, knowledge sharing, knowledge creation and identity building [30, 31]. This orientation to capacity building was a prerequisite and embraced as integral to the academic programmes at the core of teaching and learning initiatives at the UWC. It was anticipated that a multidisciplinary CoP would result in a good knowledge mix, increased social capital, tacit knowledge and a good institutional mix of disciplines. Success of such an endeavour depended on integrity, honesty and respect [8].

There was an impetus to engage with institutional policy changes that were occurring within the University as a whole and to infuse educational and capacity-building components to develop a more coherent approach to the teaching and learning of health ethics among students and academic faculties in the health sciences. An initiative to build competence within a CoP around health ethics in health research, education and clinical care was thus begun partly with the view from the HPCSA [28]. The anticipated outcomes were the following: (1) to articulate an understanding of moral reasoning and the philosophical foundations of ethical thinking; (2) to apply ethical principles of care to a variety of clinical situations; (3) to incorporate key elements of ethical health research practice to write, review or otherwise adjudicate research protocols; (4) to apply ethical principles to public health policy and practice scenarios, and (5) to apply ethical principles to health personnel education practice.

There were two main beneficiaries of this initiative. The first was the students and the second the academic faculties engaged in health research across a variety of different areas, e.g. technical improvements in the delivery of care, the improvement of materials, the better understanding and the genetics of various types of diseases, and the delivery of more effective public programmes for the prevention of such diseases. The proposed initiative addressed the content and instructional design of undergraduate and postgraduate curricula and established the tools for delivery to on-site and distance-learning students. The project was built upon existing UWC infrastructure for e-learning, proven text and case-based learning formats and face-to-face encounters, as well as emerging interactive technologies to enhance the learning process in the undergraduate and postgraduate curriculum of health sciences education.

Students who have early clinical experience and integration with classroom instruction, which is prevalent in problem-based learning environments, appear to have superior skills in social and cognitive performance [32]. If students are to develop critical thinking skills through either case-based or problem-based learning, however, then formidable challenges remain and include the diversity of a multinational class, an approach that can cross multiple disciplines, a focus on material that more effectively translates into clinical practice, and a structure that makes students less instructor dependent and more proactive in their own learning. An educator should thus remain only as a facilitator $[33,34]$.

\section{The Realization of an Innovative Healthcare Curriculum}

It was essential to build on pre-existing capabilities by linking the disciplines with different strengths and experiences and to ensure unity amid diversity. Over a period of a year, a CoP evolved that developed a comprehensive framework to guide activities, specifying outputs, outcomes and impacts. It was anticipated that the established $\mathrm{CoP}$ would be a conduit to engage with the structures that had already been established and implemented according to the University Research Ethics Policy [35]. Institutional policies and practices influence the delivery of healthcare to the community. The principles of bioethics are dynamic and reinforce each other. These principles are powerful tools that help reshape existing policies or develop new ones.

The innovative character of the intervention was recognized; therefore, explicit focus was applied to observe whether synergies could be established to reinforce institutional processes and structures capable of effectively and sustainably achieving desired outcomes and impacts. What was at stake was the building of a CoP among the various healthcare disciplines within the UWC to consolidate the institutional entities capable of knowledge translation in order to improve ethics and research ethics within the disciplines. 
Initially, faculties were hesitant to adopt the CoP model. Thus, there were major drawbacks that had to be overcome in the formulation of the healthcare curriculum and the teaching of ethics. First, an interdisciplinary approach required forsaking previously established boundaries between disciplines and individual comfort zones. Second, this transition demanded openness to criticism, the willingness to admit ignorance in certain disciplines, and the eagerness to face novel challenges in the management and transfer of knowledge. Third, additional training in bioethics was needed to apply principles across traditionally compartmentalized disciplines, i.e. medicine, law and human rights, dentistry, pharmacy, nursing and global public health, to generate ideas towards curriculum reform. If ethics were focused principally on professional conduct and jurisprudence within the healthcare curriculum and individuals remained comfortable only at that level, then further innovative reform would not be realized. Lastly, an interdisciplinary approach required students to understand the relevance of certain disciplines from one year to the next. Otherwise, students' academic perception would be such that those disciplines may be useless to academic achievement in consecutive years [36]. In spite of these limitations and initial drawbacks, CoP is self-perpetuating, as it generates social capital and fosters collaboration to draw more people into participation [19]. CoPs may thus overcome the establishment's initial reticence to implement ethical practices and teaching into an innovative healthcare curriculum. To monitor progress, documentation of the processes, activities and participant contributions were and will be consistently maintained.

The domain focus was the improvement of health ethics teaching. The initial phase consisted of participatory interaction to achieve consensus on the components needed for a comprehensive health ethics framework that incorporated health research, education and clinical care. The innovative proposed interactive framework consisted of ethical and legal issues pertinent to care and research that could be integrated and inserted into the curricular structure of several disciplines, at either the undergraduate or postgraduate level, and assessed throughout the curriculum.

Underpinning the exercise of building a CoP was the understanding that ethics is essential for the provision of good healthcare, education to healthcare workers, health research, and global and public health. Fundamental to this practice would be a knowledge of the philosophical foundations of ethics that would provide the basis for moral reasoning as it applies to healthcare, education, re-

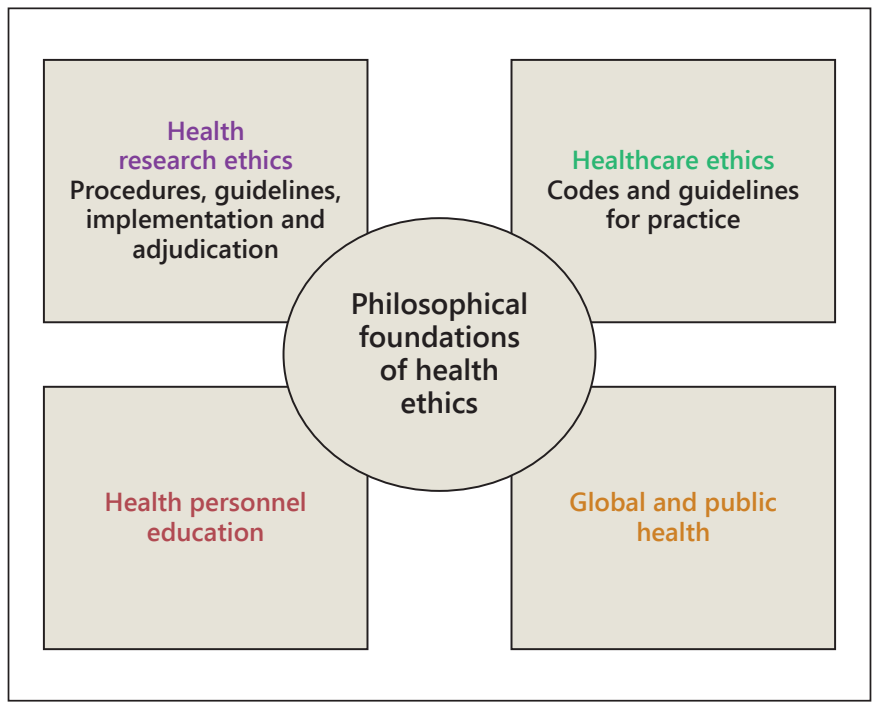

Fig. 1. The centrality of the philosophical foundations of health ethics.

search and public health $[4,5,37]$. It will thus (1) compare different philosophical viewpoints on ethics, morality and justice; (2) provide language to interrogate different social value systems; (3) enable the critical evaluation of competing notions of rights, and (4) translate into guidelines and concepts that will provide sturdy ethical tools and frameworks in healthcare. Philosophical foundations of health ethics are central to and intersect with the four key areas: health research, health personnel education, healthcare, and global and public health (fig. 1).

\section{Designing Component Parts of the Curriculum}

The design and components of this innovative curriculum can be described only qualitatively at its current level of development (fig. 1). This should not be interpreted as a limitation but a framework for future work. Hence, specific assessment tools that measure outcomes, e.g. translation into codes, guidelines and protocols, must then be further developed to determine the final validity of the model or whether revision is still needed.

\section{Health Research Ethics}

The health research ethics component will assist faculties and students in their administrative commitments and has implications for ensuring due process is carried out with regard to health research procedures. Specific 
Table 1. Key topics in healthcare ethics

(1) Informed consent and refusal to treat

(2) The clinical relationship, truthfulness, trust and good communication

(3) Confidentiality and good clinical practice

(4) Health research

(5) The new genetics/genomics/individual tailored therapy

(6) Treating children

(7) Care for those with mental disorders and disabilities

(8) Human reproduction

(9) Resource allocation and issues of justice

(10) Human rights

(11) Capacity building for ethical health care

(12) Vulnerabilities created by duties of healthcare workers

(13) Life, death, dying and euthanasia

Adapted from Doyal and Gillon [41].

guidelines and codes are provided to enable the implementation of ethical health research. It is anticipated that the earlier first, second and third years of study will focus on the basic ethical tenets of informed consent, confidentiality, truth-telling, resource allocation, and tensions between moral focus on individual interest versus the common good, along with the philosophical underpinnings of related debates. This core of ethical and legal understanding in health research will continue in the later fourth and fifth years and emphasis will be placed on analysing the impact of socio-cultural differences and the impact that such differences can have on professional standards. During this period, students will have an opportunity to constitute their own ethics committee, supervised by the appropriate faculty. Equally important, each individual student will work with a faculty researcher in preparing a protocol for evaluation by the institutional research ethics committee. All of these activities will build capacity from the beginning of their training for acceptable ethical and legal work in the future.

\section{Healthcare Ethics}

The healthcare ethics component will apply to the provision of clinical healthcare in specific ways and these will translate into the codes, guidelines and protocols related to the practice of ethical healthcare. One example of a specific and practical application from the CoP model in healthcare ethics curriculum innovation is in the clinical management of patients who are no longer legally competent. Care for patients with mental disorders and disabilities who can no longer make decisions on their own behalf

Innovative Ethics Curriculum in Healthcare present unique challenges (table 1). A patient that is brought to a dental clinic with Alzheimer's disease and is no longer legally competent will require a surrogate to make decisions. The principles of surrogacy are typically taught and applied in the intensive care unit setting or in end-of-life scenarios in medicine. However, with an interdisciplinary approach, the same principles as taught traditionally in the disciplines of medicine and law can also be taught in the dental school ethics curriculum related to the management of patients with advanced neurological diseases. That knowledge can thus be transferred into a context-based clinical experience $[9,10]$. Codes, guidelines and protocols for the management of such patients can be established and an ethical analysis and assessment can be provided in the clinical dental chart. Ethics and respect for human rights is thus fundamental to healthcare workers in their role as citizens (table 1).

\section{Health Personnel Education in Ethics}

The health personnel education in ethics component will apply to the education of the various health professions in unique ways and translate into codes and protocols for the practice of ethical healthcare. In the dental setting, for example, health personnel may include dental hygienists and dental assistants who provide clinical care. Truthfulness, trust and good communication, as well as maintaining confidentiality and good clinical practice, are fundamental to the patient-healthcare provider relationship (table 1). As also shown in figure 1, the philosophical foundations of health ethics intersect with and are thus a critical part of health personnel education in ethics. Indeed, additional personnel involved with record keeping and billing as third parties also have access to patients' private information related to healthcare. Health personnel may be aware not only of the patient's dental needs but also of any underlying systemic condition that may affect the outcome of treatment. In that regard, health personnel become part of the sphere of confidentiality and privacy equally to the dentist. The right to privacy of patients is justified by rights of autonomous choice that are correlative to the obligations expressed in the principle of respect for autonomy $[1,4]$. In that regard, respect for the autonomous wishes of patients is such that there is no intrusion in any way.

The structure of the CoP permits dialogue and the exchange of ideas coupled with experiential knowledge in the clinical setting. Thus, a dialogue between members of the dental healthcare team, third parties such as insurance or government agencies, billing and accounting services, and the community to explore, for example, the applica- 
Table 2. Key public health research topics in ethics

(1) Vulnerable communities

(2) Global health ethics

(3) Public health ethics

(4) Health and human rights

(5) Global health research agendas

(6) Capacity building for ethical research

(7) Ethics in the developing world contexts and culture

(8) Inequities in health and health research

(9) Global guidelines and processes for ethical research

(10) Local guidelines and processes

tion of the principle of autonomy and respect for persons in various scenarios can thus make the translation from theory into practical protocols and guidelines realizable for clinical practice, and these in turn can become part of an innovative curriculum for healthcare professionals.

\section{Global and Public Health Ethics}

The global and public health ethics component will apply to key areas that move from the focus of individual health to that of the public (table 2) and translates into codes and protocols for the practice of ethical public health. In that regard, a careful distinction must be maintained between individual health and the notion of individualistic autonomy from medical ethics versus population health and the wider social injustices that are responsible for health-related vulnerability and risk of disease. Failure to maintain such a distinction would constitute a basic philosophical category mistake [38].

\section{Formulation of Healthcare and Educational Policies}

Vernillo [39] commented on an ethical, legal and social implications programme for garnering the financial support of neuroscience research at governmental or national level. The lay public may be chary of supporting research that investigates the functioning of the brain and behaviour, for example, because the public does not have the technical expertise to assess the complexities of such research. The public may also view neuroscience research as highly intrusive, particularly if it enters the brain, which is often regarded as a sanctuary of thought and emotion. The public thus uses heuristics initially as cognitive short cuts to determine the worthiness of such research. Separate committees that would work together to include a panel of experts in medicine, research and bio- ethics, and another committee without particular scientific expertise, e.g. individuals in non-professional occupations, were proposed to foster dialogue at governmental level, thus generating robust debate and potentially reshaping research policy [39].

How then might the CoP analogously influence healthcare or educational policies at governmental or university level? An approach is to organize two committees at governmental level to implement changes in healthcare and educational policies, namely, (1) a panel of experts to include healthcare providers, health policy makers, government officials, bioethicists and healthcare educators and (2) members of the lay public such as journalists, writers and social commentators, those in non-professional occupations, and students at university and professional schools. The social capital that may be generated could lead to the formulation of policies in healthcare and healthcare education.

In the university environment, the CoP model could be applied whereby a committee of experts such as healthcare professionals, public health policy makers, bioethicists, scientists, chairpersons of the individual departments, and administrators represent one committee. That committee could be the core group and may consist of comparably equal numbers from each representative area, e.g. dentistry, medicine, law, public health, bioethics and research. A second committee as a smaller active group working in concert with the larger core group could represent professional educators from the different clinical and basic science departments, student representatives from each of their respective professional/university classes, and select members from the community to include the lay public and patients with a vested interest in educational reform in the healthcare professions. The members of the active group, particularly the students as consumers of the innovative curriculum, are those who would most likely benefit considerably. Although the precise number of members for the core and active groups are not yet determined, the goal would be to ensure productivity by avoiding groups that become unwieldy. The core and active groups would be able to generate collaborations and exchange ideas to reshape educational curriculum and healthcare policy.

\section{Conclusion}

In many ways it is premature to pass judgment on the exercise described in this paper regarding its effectiveness and the sustainability of the CoP, which was developed as 
part of an educational curriculum model for healthcare in allied professions to include nursing and dentistry. There are major drawbacks and challenges using the CoP model for the reform of the professional school ethics curriculum. Certain examples at governmental and university levels are proposed that may effectuate healthcare policy and professional healthcare curriculum innovation and possibly extend to the conduct of research in developing countries. Indeed, the CoP has its origins perhaps comparatively distant from healthcare in the fields of cognitive anthropology and business $[7,8]$.Therefore, specific assessment tools that measure outcomes in an innovative healthcare curriculum must be developed and implemented. The CoP at UWC was fundamentally self-established whereby members recognized that it was their own domain and practice that brought them together to form the basis of a community. It has been reported that 'great effort is required to create a functioning multidisciplinary CoP' [40]. Some of the challenges included the need to pay attention to maintaining the team and its identity, valuing diversity, and finding dedicated time for team members to reflect, plan and work together across inter- disciplinary boundaries. Nonetheless, the initiative has vividly demonstrated how interdisciplinary, interfaculty alliances and collaboration offer promising ways to strengthen capacity and learn together about ways to continually improve teaching and learning in health ethics. Assessing the legacy of the process will require long-term monitoring of the results, challenges and sustainability. However, we do feel strongly that the consolidation of a $\mathrm{CoP}$ predicts the core values and vision that was the original goal of our endeavour.

\section{Acknowledgements}

We wish to thank the Fogarty International Centre, Professor Solly Benatar and colleagues who participated in the workshops: Neil Myburgh, Nadine Butler, Greta Geerts, Gail Hughes, Chrisleen Rayner and Christina Zarowsky.

\section{Disclosure Statement}

The authors have no conflicts of interest to disclose.

\section{References}

1 Lo B: Resolving Ethical Dilemmas: A Guide for Clinicians, ed 4. New York, Walters Kluwer Health/Lippincott, Williams \& Wilkins, 2009.

2 Ozar DT, Sokol DJ: Dental Ethics at ChairSide Professional Principles and Practical Applications, ed 2. Washington, Georgetown University Press, 2002, pp 43-62.

3 Rule JT, Veatch RM: Ethical Questions in Dentistry, ed 2. Quintessence, 2004.

4 Beauchamp TL, Childress JF: Principles of Biomedical Ethics, ed 6. New York, Oxford University Press, 2009, pp 317-323.

5 World Health Organization: Casebook on Ethical Issues in International Research. http://whqlibdoc.who.int/publications/2009/ 9789241547727_eng.pdf(accessed August 19, 2012).

6 Lave J, Wenger E: Situated Learning: Legitimate Peripheral Participation, ed 1. Cambridge, Cambridge University Press, 1991, p 29.

7 Wenger E: Communities of Practice: Learning, Meaning, and Identity, ed 1. Cambridge, Cambridge University Press, 1998, pp 72-73.

8 Wenger E, McDermott R, Snyder WM: Cultivating Communities of Practice, ed 1. Boston, Harvard Business School Press, 2002, p 150.

$\checkmark 9$ Wasko M, Faraj S: It is what one does: why people participate and help others in electronic communities of practice. J Strategic Inf Syst 2000;9:155-173.
10 Hildreth P, Kimble C: Knowledge Networks: Innovation through Communities of Practice, ed 1. Hershey, Idea Group, 2004, pp 153158.

11 Hildreth P, Kimble C: The duality of knowledge. Inform Res 2002;8:52-58.

12 Davenport TH, Prusak L: Working Knowledge. How Organizations Manage What They Know, ed 2. Cambridge, Harvard Business School Press, 2000, p 240.

13 McDermott R, Archibald D: Harnessing Your Staff's Informal Networks. Harvard Business Review 2010. http://hbr.org/2010/03/harnessing-your-staffs-informal-networks/ar/1 (accessed August 19, 2012).

14 Kimble C, Hildreth P, Bourdon I: Communities of Practice: Creating Learning Environments for Educators, ed 1. Charlotte, Information Age Publishing, 2008.

15 Thomas JC, Kellogg WA, Erickson T: The knowledge management puzzle: human and social factors in knowledge management. IBM Syst J 2001;40:863-884.

16 Lesser E: Leveraging social capital in organizations; in Lesser EL (ed): Knowledge and Social Capital. Boston, Butterworth-Heinemann, 2000, pp 3-16.
17 Seely Brown J, Duguid P: Organizational learning and communities-of-practice: toward a unified view of working, learning and innovation. Organ Sci 1991;2:40-57.

18 Capelli P: A market-driven approach to retaining talent. Harv Bus Rev 2000;78:103-111.

19 Lesser EL, Storck J: Communities of practice and organisational performance. IBM Syst J 2001;40:831-841.

20 Dalkir K: Knowledge Management in Theory and Practice, ed 1. Burlington, ButterworthHeinemann, 2005, pp 19-20.

21 Duguid P: The art of knowing: social and tacit dimensions of knowledge and the limits of the community of practice; in Sawhney $\mathrm{H}$ (ed): The Information Society. New York, Taylor \& Francis, 2005, pp 109-118.

-22 Ardichvilli A, Page V, Wentling T: Motivation and barriers to participation in virtual knowledge sharing in communities of practice. J Knowledge Manag 2003;7:64-77.

$23 \mathrm{Tu} \mathrm{C}-\mathrm{H}$ : The management of social presence in an online learning environment. Int J ELearning 2002:34-45.

24 Bate SP, Robert G: Knowledge management and communities of practice in the private sector: lessons for modernizing the National Health Service in England and Wales. Public Admin 2002;80:643-663.

25 Coghlan D, Brannick T: Doing Research in Your Own Organisation, ed 2. London, Sage Publications, 2005.
Innovative Ethics Curriculum in Healthcare 
26 Fitzgerald L, Dopson S: Professional boundaries and the diffusion of innovation; in Dopson S, Fitzgerald L (eds): Knowledge to Action? Evidence-Based Health Care in Context. Oxford, Oxford University Press, 2006, pp 104-131.

-27 Sveiby KE, Simon R: Collaborative climate and effectiveness of knowledge work - an empirical study. J Knowledge Manag 2002;6: 420-433.

28 HPCSA, Health Professions Council of South Africa: Protecting the Public and Guiding the Professions. http://www.hpcsa.co.za/ (accessed August 18, 2012).

29 Gustavsson B: Revisiting the philosophica roots of practical knowledge; in Higgs J, Richardson B, Abrandt Dahlgren M (eds): Developing Practice Knowledge for Health Professionals. Edinburgh, Butterworth-Heinemann, 2004, pp 35-50.
30 Li LC, Grimshaw JM, Nielsen C, et al: Use of communities of practice in business and health care sectors: a systematic review. Implement Sci 2009;4:16.

31 Cox A: What are communities of practice? A comparative review of four seminal works. J Inform Sci 2005;31:527-540.

32 Neville AJ: Problem-based learning and medical education forty years on. A review of its effects on knowledge and clinical performance. Med Princ Pract 2009;18:1-9.

33 Shankar PR: Problem-based learning: the right direction for medical teaching? Med Princ Pract 2008; 17:171-172.

34 Robinson DL: The reform of medical education. Med Princ Pract 2007;16:467-468.

35 University of the Western Cape University Research Policy 2008. http://www.uwc.ac.za/ index.php? module $=\mathrm{cms} \&$ action $=$ showfulltex t\&id=gen11Srv7Nme54_2067_1239789821\& parent=gen11Srv7Nme54_6363_1239265491 (accessed August 19, 2012).

36 Bouhaimed M, Thalib L, Doi SA: Perception of the educational environment by medical students undergoing a curricular transition in Kuwait. Med Princ Pract 2009;18:204-208.
Azetsop J, Rennie S: Principalism, medical individualism, and health promotion in resource-poor countries: can autonomy-based bioethics promote social justice and population health? Philos Ethics Humanit Med 2010; 5:1.

38 Munthe C: The goals of public health: an integrated, multidimensional model. Public Health Ethics 2008;1:39-52.

39 Vernillo A: An ethical, legal, and social implications (ELSI) program for neuroscience: heuristics and the lay public. AJOB Neurosci 2010;1:15-16.

40 Dopson S, Fitzgerald L: Knowledge to action? Evidence-based health care in context. Oxford, Oxford University Press, 2006, p 125.

41 Doyal L, Gillon R: Medical ethics and law as a core subject in medical education: a core curriculum offers flexibility in how it is taught but not that it is taught. BMJ 1998;316:16231624. 